\title{
A Combined Model-Order Reduction and Deep Learning Approach for Structural Health Monitoring Under Varying Operational and Environmental Conditions $^{\dagger}$
}

\author{
Matteo Torzoni ${ }^{1, *,(1)}$, Luca Rosafalco ${ }^{1,(1)}$ and Andrea Manzoni ${ }^{2,(1)}$ \\ 1 Dipartimento di Ingegneria Civile ed Ambientale, Politecnico di Milano, Piazza L. da Vinci 32, 20133 Milano, \\ Italy; luca.rosafalco@polimi.it \\ 2 MOX, Dipartimento di Matematica, Politecnico di Milano, Piazza L. da Vinci 32, 20133 Milano, Italy; \\ andrea1.manzoni@polimi.it \\ * Correspondence: matteo.torzoni@polimi.it \\ + Presented at the 7th Electronic Conference on Sensors and Applications, 15-30 November 2020; Available \\ online: https://ecsa-7.sciforum.net/.
}

Published: 15 November 2020

\begin{abstract}
The aging, deterioration and failure of civil structures are nowadays challenges of paramount importance, increasingly motivating the search of advanced Structural Health Monitoring (SHM) tools. In this work, we propose a SHM strategy for online structural damage detection and localization, combining Deep Learning (DL) and Model-Order Reduction (MOR). The developed data-based procedure is driven by the analysis of vibration and temperature recordings, shaped as multivariate time series and collected on the fly through pervasive sensor networks. Damage detection and localization are treated as a supervised classification task considering a finite number of predefined damage scenarios. During a preliminary offline phase, for each damage scenario, a collection of synthetic structural responses and temperature distributions is numerically generated through a physics-based model. Several loading and thermal conditions are considered thanks to a suitable parametrization of the problem, which controls the dependency of the model on operational and environmental conditions. Because of the huge amount of model evaluations, MOR techniques are employed to relieve the computational burden associated to the dataset construction. Finally, a deep neural network, featuring a stack of convolutional layers, is trained by assimilating both vibrational and thermal data. During the online phase, the trained DL network processes new incoming recordings to classify the actual state of the structure, thus providing information about the presence and the localization of the damage, if any. Numerical performances of the proposed approach are assessed on the monitoring of a two-storey frame under low intensity seismic excitation.
\end{abstract}

Keywords: structural health monitoring; model order reduction; deep learning; damage localization; vibration monitoring; environmental effects.

\section{Introduction}

Modern societies are strongly dependent on the use of complex structures. Since an early detection of structural faults can greatly reduce the maintenance cost over time and prevent catastrophic events [1], being able to keep civil constructions safe and reliable is fundamental for the community welfare [2]. For these reasons, in the last decades civil engineering has focused on Structural Health Monitoring (SHM) [3], aimed at detecting, localizing and quantifying damage occurrence. Especially data-driven approaches $[4,5]$ are becoming more and more widespread thanks to their capacity of easily manage the large amount of data, acquired through pervasive sensor networks. In particular, by processing 
raw vibrational signals (e.g., acceleration recordings shaped as multivariate time series), they can extract useful features, to determine the damage state of the structure. To this aim, Deep Learning (DL) algorithms can automatically extract damage-sensitive features [6] and relate them with the corresponding structural states, by exploiting temporal correlations within and across time recordings.

Considering a finite number of predefined damage scenarios, the detection and localization of damage in structures can be treated within a classification framework. By employing a supervised classifier, the goal is to predict the categorical class (i.e., a label referring to a predefined damage scenario) of new incoming data, on the basis of past observations. Supervised techniques require labeled data on the possible damage conditions of the structure, which however are hard (if not impossible) to acquire for civil applications. This drawback calls for a Simulation-Based Classification (SBC) $[1,7,8]$, to numerically simulate the effect of damage on the structural response. In such hybrid model-data SHM approach, a dataset of synthetic time-signals, accounting for relevant operational conditions and varying environmental effects, is generated through simulations of a physics-based model, for the whole set of considered damage scenarios, and thus assimilated with the DL algorithm.

In order to replace the expensive numerical models, relying on the Finite Element (FE) method, a Model Order Reduction (MOR) strategy, such as the Reduced Basis (RB) method [9,10], can be adopted to accelerate the dataset construction.

The proposed methodology exploits an offline-online decomposition. During the preliminary offline phase, our DL-based classifier is trained on a numerically pre-built dataset of labeled inputs; during the online phase, the trained classifier processes unseen experimental signals acquired on the fly, returning as output the structural state which might have most likely produced them. Our classifier is based on a Fully Convolutional Network (FCN) architecture, already successfully applied in [8,9].

As well as damage phenomena, environmental conditions could affect measured signals. Thermal fluctuations (both daily and seasonal) can influence a wide range of material properties and induce structural displacements. It is not easy to distinguish these effects from those of damage. For this reason, thermal effects are simulated in the numerical model of the structure and temperature measurements are used together with vibrational ones as inputs to the classifier.

\section{SHM Methodology: Dataset Definition and Damage Classifier}

Considering an observation windows $(0, T)$, short enough to assume frozen operational, environmental and damage conditions, the damage state of the structure is monitored by collecting vibrational and temperature data through a sensor network featuring $N_{u}$ vibrational sensors, with fixed sampling period $\Delta t$, and $N_{\phi}$ thermometers. The network arrangement has been designed starting from an initial placement involving a high number of sensors, progressively reduced evaluating the classifier performances on multiple datasets generated according to different sensor configurations. Vibrational recordings consist of displacement and/or acceleration measurements $\mathbf{u}_{n}, \ddot{\mathbf{u}}_{n}\left(n=1, \ldots, N_{u}\right)$ of length $L=T / \Delta t$ (for sake of simplicity, in this section we only consider displacement measurements), while each thermometer outputs a single value $\phi_{n}\left(n=1, \ldots, N_{\phi}\right)$. A single data instance is composed of a set of displacement recordings $\mathbf{U}_{i}\left(\boldsymbol{\eta}_{u}^{i}, \eta_{\phi}^{i}, g_{i}\right)=\left[\mathbf{u}_{1}^{i}, \ldots, \mathbf{u}_{N_{u}}^{i}\right] \in \mathbb{R}^{L \times N_{u}}$ and temperature measurements $\boldsymbol{\Phi}_{i}\left(\boldsymbol{\eta}_{\phi}^{i}\right)=\left(\phi_{1}^{i}, \ldots, \phi_{N_{\phi}}^{i}\right) \in \mathbb{R}^{N_{\phi}}$, where: $g_{i}$ labels the damage state undergone by the structure in the $i$-th instances, modeled as a localized stiffness reduction in pre-designated regions; $\eta_{u}^{i}$ and $\eta_{\phi}^{i}$ label the set of parameters controlling the mechanical and the temperature field, respectively. The dataset $\mathbf{D}$ is made of $I$ instances $\left\{\mathbf{U}_{i}, \boldsymbol{\Phi}_{i}\right\}, i=1, \ldots, I$; to relieve the computational burden of its generation, the Full-Order Model (FOM), relying on the FE method, is replaced by a Reduced-Order Model (ROM).

According with the adopted classification framework, only a discrete number of damage scenarios $g=0, \ldots, G$ has to be defined on the basis of the mechanical behavior, load conditions and aging processes interesting similar structures [4]; the baseline undamaged state is labeled as $g=0$.

A continuous probability density function (pdf) describes the occurrence of each entry of $\boldsymbol{\eta}_{u}^{i}$ and $\boldsymbol{\eta}_{\phi}^{i}$, while a discrete pdf governs the occurrence of $g_{i}$. The parameter set $\eta_{\phi}^{i}$, sampled from pdfs taking into account the locality of interest and the seasonality of temperature fluctuations, controls the 
temperature profiles imposed at the domain edges. The set $\boldsymbol{\eta}_{u}^{i}$ parametrizes the external loads (e.g., amplitude and frequency of a dynamical load) and the damage level $\delta_{i}$, intended as the intensity of the stiffness reduction involving the subdomain related to $g_{i}$. A suitable sampling rule (e.g., a Latin Hypercube Sampling) has been adopted to explore the parametric space defined combining $\boldsymbol{\eta}_{u^{\prime}}, \boldsymbol{\eta}_{\phi}$ and g. Each sampling $\left\{\boldsymbol{\eta}_{u}, \boldsymbol{\eta}_{\phi}, g\right\}_{i}$ uniquely identifies the corresponding dataset instance $\left\{\mathbf{U}_{i}, \boldsymbol{\Phi}_{i}\right\}$.

Once built, $\mathbf{D}$ is employed to train and validate a classifier $\mathcal{G}$. During the training phase, $I_{t r}$ instances are employed by $\mathcal{G}$ to learn the underlying mapping between the $i$-th instance $\left\{\mathbf{U}_{i}, \boldsymbol{\Phi}_{i}\right\}$ and $g_{i}$, while $I_{\text {val }}$ instances (with $I=I_{t r}+I_{\text {val }}$ ) are used to validate the learning process. Once trained, $\mathcal{G}$ should be able to correctly map an unseen instance $\left\{\mathbf{U}_{i}, \boldsymbol{\Phi}_{i}\right\}$ into the correct damage state $g_{i}$. In absence of experimental data, the generalization capabilities have been assessed on a test set built through FOM simulations, in this way ensuring a better fidelity to the experimental framework. For sake of clarity, from now on we disregard to specify the instance index $i$.

Our FCN architecture, resembling the one proposed in [8], process $\mathbf{U}$ by exploiting a stack of three Convolutional Units (CU) followed by a Global Average Pooling (GAP), whose output is merged with $\boldsymbol{\Phi}$ through a Concatenation Block (CB). These latter support the classifier in recognizing thermal effects of material contraction/expansion and stiffening/softening within the observed dynamics, to not confuse the environmental variability with damage [11]. Thus, a dense layer operate a linear mapping, ruled by a weight matrix $\Theta$, allowing a final Softmax layer to perform the classification task. Each of the three CUs is formed by a Convolutional Layer (CL), together with Batch Normalization, to stem gradient instability issues during training, and ReLU activation function. In a CL, connection weights $\Omega$ can be imagined as filters of kernel size $H_{j}$, with $j=1,2,3$, to be applied to the output of the previous layer. Each convolutional layer applies $N_{j}$ filters to its inputs, yielding an output made of $N_{j}$ feature maps. This composition of nonlinear transformations, makes each damage target class linearly separable and allows to address the temporal pattern recognition, exploiting inter-sensor correlations, by simultaneously analyzing $\mathbf{U}$. The resulting feature maps are condensed by the GAP, which outcomes a single, yet highly informative, description of its input channel $\mathbf{u}_{1}, \ldots, \mathbf{u}_{N_{u}}$.

While training $\mathcal{G}$, the learning algorithm tunes the weights $\Omega$ and $\Theta$, by iteratively minimizing a loss function over the $I_{t r}$ instances. As usually done in classification frameworks, the adopted loss function is the cross entropy; Adam, a first-order stochastic gradient descend algorithm, has been employed to perform the iterative minimization process. At each iteration, a certain number of instances $B$, called mini-batch, are analyzed simultaneously to update the connection weights; each time all the $I_{t r}$ instances have been processed is said to be an epoch.

The FCN hyperparameters $\left(N_{j}, H_{j}, B, I, \mathrm{n}^{\circ}\right.$ epochs), initially set according to [8], have been tuned through the repeated evaluation of the classification accuracy. Here we have adopted: $N_{1}=16$, $N_{2}=32$ and $N_{3}=16$, as number of filters; $H_{1}=8, H_{2}=5$ and $H_{3}=3$, as kernel sizes; $B=16$ as mini-batch size; $I=15000$, as number of instances, with ratio $I_{t r}: I_{v a l}=75: 25 ; 1000$ training epochs.

\section{SHM Methodology: Dataset Population}

Adopting a SBC approach, the generation of the $I$ instances has been carried out by evaluating the physics-based model of the structure, for multiple values of the input parameters $\eta_{u}$ and $\eta_{\phi}$ for each considered scenarios $g=0, \ldots, G$.

The thermo-mechanical behavior of the structure has been modeled through the standard linear thermo-elasticity theory employing a one-way coupling approach; the thermal field is determined independently from the kinematic one, but still influences the material deformations. Moreover, having supposed monitoring windows of fixed duration, significantly lower than the time required to experience notable temperature excursions, the thermal field has been evaluated disregarding its temporal dependence. To reflect an oscillation of the material temperature into the dynamical response, a local dependency of the Young modulus $E$ on temperature has been introduced. Lastly, due to the small relevance in the identification of continuously excited dynamic systems [12,13], damping effects 
have been disregarded; besides, modeling the structural damage as a selective reduction in stiffness, frozen in $(0, T)$, the mechanical behavior has been treated as linear [3]. The FOM reads as follows:

$$
\mathbf{K}_{\varphi} \boldsymbol{\varphi}=\mathbf{f}_{\varphi}
$$

$$
\left\{\begin{array}{l}
\mathbf{M}_{v} \ddot{\mathbf{v}}(t)+\mathbf{K}_{v} \mathbf{v}(t)=\mathbf{G}_{v} \boldsymbol{\varphi}+\mathbf{f}_{v}(t), \quad t \in(0, T) \\
\mathbf{v}(0)=\mathbf{v}_{0} \\
\dot{\mathbf{v}}(0)=\dot{\mathbf{v}}_{0}
\end{array},\right.
$$

and results from a Galerkin-FE discretization of a stationary diffusion problem and of a elasto-dynamic problem with coupling term, respectively. In particular: $t \in(0, T)$ denotes the time coordinate; $\boldsymbol{\varphi}=\boldsymbol{\varphi}\left(\boldsymbol{\eta}_{\phi}\right) \in \mathbb{R}^{M_{\varphi}}$ is the temperature vector; $\mathbf{K}_{\varphi} \in \mathbb{R}^{M_{\varphi} \times M_{\varphi}}$ is the thermal conductivity matrix; $\mathbf{f}_{\varphi}=\mathbf{f}_{\varphi}\left(\boldsymbol{\eta}_{\phi}\right) \in \mathbb{R}^{M_{\varphi}}$ is the thermal right hand side vector; $\mathbf{v}=\mathbf{v}\left(t, \boldsymbol{\eta}_{u}, \boldsymbol{\varphi}, g\right) \in \mathbb{R}^{M_{v}}$ is the displacement vector; $\mathbf{M}_{v} \in \mathbb{R}^{M_{v} \times M_{v}}$ is the mass matrix; $\mathbf{K}_{v}=\mathbf{K}_{v}(\boldsymbol{\varphi}, g) \in \mathbb{R}^{M_{v} \times M_{v}}$ is the elastic stiffness matrix; $\mathbf{G}_{v} \in \mathbb{R}^{M_{v} \times M_{\varphi}}$ is the coupling term; $\mathbf{f}_{v}=\mathbf{f}_{v}\left(t, \boldsymbol{\eta}_{u}\right) \in \mathbb{R}^{M_{v}}$ is the mechanical right hand side vector; $M_{\varphi}$ and $M_{v}$ denote, respectively, the number of degrees of freedom (dofs) of the temperature and displacement FE spaces.

$\mathrm{Pb}$. (1) is first solved to determine the temperature field $\varphi$. Vector $\varphi$ is transformed into equivalent nodal forces through $\mathbf{G}_{v}$. A displacement field, compatible with $\varphi$, is determined by solving a static mechanical problem under the coupling action only, and is assumed as the reference around which the dynamics governed by $\mathrm{Pb}$. (2) oscillates. Discretization in time has been made according to the sensors sampling rate. For the integration in time of $\mathrm{Pb}$. (2) we have exploited a generalized- $\alpha$ method.

The FOM relies upon a number of dofs $M_{v}$ and $M_{\varphi}$ depending on the adopted (potentially fine) discretization. The RB method, used to construct the dataset $\mathbf{D}$, exploits a Galerkin-Proper Orthogonal Decomposition (POD) ROM, whose POD basis is built starting from a set of FOM solutions (snapshots), computed within the prescribed parameters range. The ROM solution is then sought by solving the reduced-order problem resulting from the Galerkin projection of the FOM onto the reduced basis.

Displacements and temperatures related to the $i$-th sampling $\left\{\boldsymbol{\eta}_{u^{\prime}}, \boldsymbol{\eta}_{\phi}, g\right\}$ are time integrated and collected in $\mathbf{V}=\left[\mathbf{v}_{1}, \ldots, \mathbf{v}_{L}\right] \in \mathbb{R}^{M_{v} \times L}$ and $\boldsymbol{\varphi}=\left(\varphi_{1}, \ldots, \varphi_{M_{\varphi}}\right)^{\top} \in \mathbb{R}^{M_{\varphi}}$, respectively. The monitored dofs $\mathbf{U}$ and $\boldsymbol{\Phi}$, mimicking the sensors recordings, are extracted through two boolean matrices $\mathbf{T}_{u} \in \mathbb{R}^{N_{u} \times M_{v}}$ and $\mathbf{T}_{\phi} \in \mathbb{R}^{N_{\phi} \times M_{\varphi}}$, as $\mathbf{U}=\left(\mathbf{T}_{u} \mathbf{V}\right)^{\top}$ and $\mathbf{\Phi}=\left(\mathbf{T}_{\phi} \boldsymbol{\varphi}\right)^{\top}$.

\section{Numerical Test Case}

The proposed approach has been assessed on the monitoring of the 2D frame depicted in Fig. 1. Considering a structural thickness of $0.1 \mathrm{~m}$, the plane stresses formulation has been adopted. The bottom edges are assumed perfectly clamped to the ground. The geometry has been discretized in 2938 constant strain triangle finite elements. The adopted mechanical and thermal properties are those of an ordinary reinforced concrete: Young modulus $E=30 \mathrm{GPa}$; Poisson ratio $v=0.2$; density $\rho=2500 \mathrm{~kg} / \mathrm{m}^{3}$; thermal expansion coefficient $\alpha_{L}=12 \cdot 10^{-6}{ }^{\circ} \mathrm{C}^{-1}$; stiffness thermal coefficient $\alpha_{E}=4.5 \cdot 10^{-4}{ }^{\circ} \mathrm{C}^{-1}$. The last two proprieties allow to relate the local material temperature to the thermally induced anelastic deformations and to the material stiffening/softening, respectively.

The structure is excited by low intensity seismic loads. To this aim, we have employed the empirical equations for predicting the attenuation of ground motion proposed in [14] and implemented in [15]. The main advantage of this tool is the possibility to generate random spectrum-compatible accelerograms as function of: local magnitude $Q$; epicentral distance $R$; site geology. To ensure the structure to behave in elastic regime, those parameters have been limited within the following ranges: $Q \in(4.8,5.3) ; R \in(80,100) \mathrm{km}$; rocky conditions. The parameters $Q$ and $R$ have been described by two uniform pdfs $\mathcal{U}_{Q}(4.8,5.3)$ and $\mathcal{U}_{R}(80 \mathrm{~km}, 100 \mathrm{~km})$, respectively.

Nine damage scenarios have been simulated by means of a localized stiffness reduction. Each structural state $g \in\{1, \ldots, 8\}$ identifies a structural damage occurring in the subdomain $\Omega_{s}, s=1 \ldots, 8$, respectively; the damage-free baseline is labeled as $g=0$. The occurrence of damage scenarios has been modeled with a (discrete) uniform $\operatorname{pdf} \mathcal{U}_{g}(\{0, \ldots, 8\})$ describing $g$. The damage level $\delta$, representing the intensity of the stiffness reduction to be applied, has been modeled by a uniform $\operatorname{pdf} \mathcal{U}_{\delta}(5 \%, 25 \%)$. 


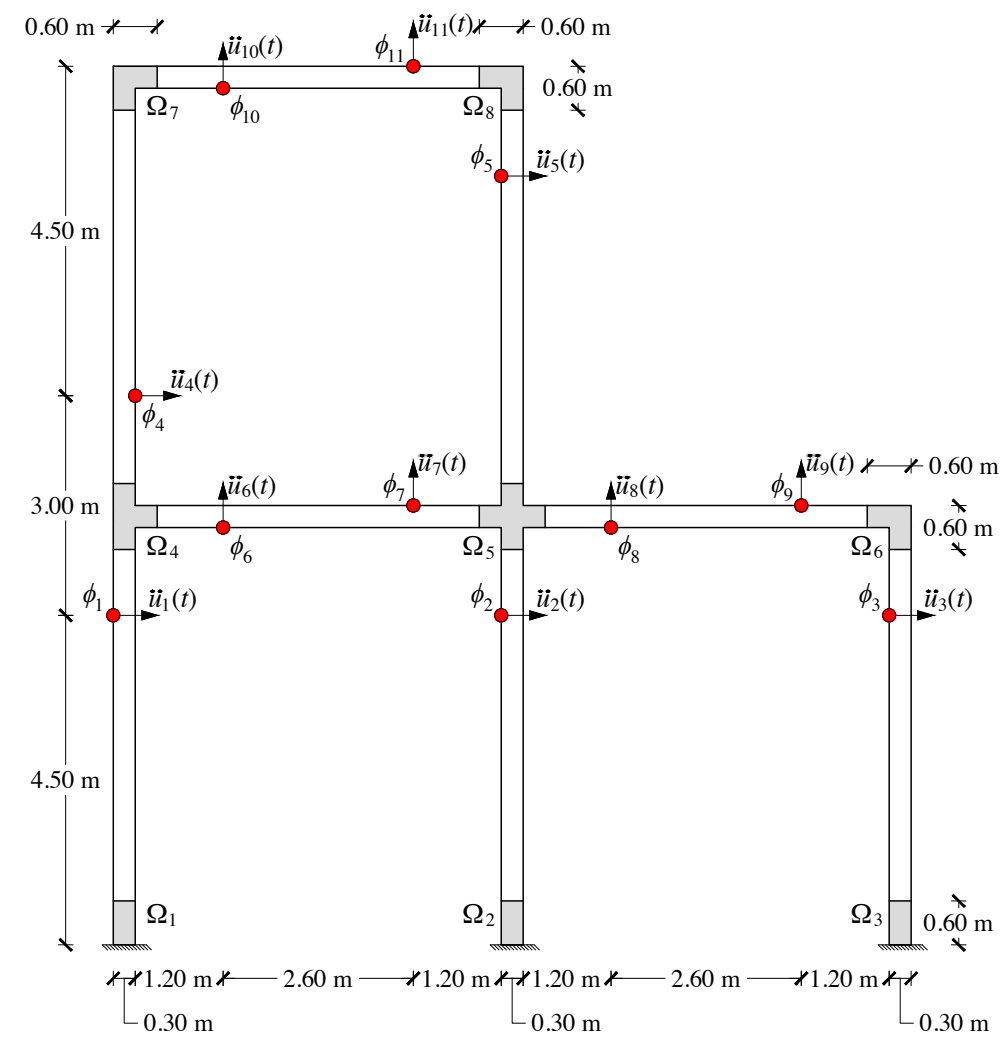

Figure 1. 2D frame configuration, considered damage scenarios, sensor network arrangement.

To simulate potential thermal distributions, resembling those experienced by the structure, $\mathrm{Pb}$. (1) is solved under the action of thermal profiles imposed on all edges. Those profiles have been modeled by interpolating temperature values at edges midpoints and corners, which are nothing but the components of the parameter vector $\eta_{\phi}$. Temperatures have been assumed to be constant and equal for the three edges in contact with the ground, while parabolic profiles have been used elsewhere. In this way, a total of 28 parameters $\eta_{\phi}^{l}$ (with $\left.l=1, \ldots, 28\right)$ are involved. From the temperature data of the city of Milan, a Gaussian pdf $\mathcal{N}_{m}\left(\mu_{m}, \sigma_{m}\right), m=1, \ldots, 12$, has been defined for each month, being $\mu_{m}$ and $\sigma_{m}$ the monthly average and standard deviation, respectively. Each time $\mathrm{Pb}$. (1) is solved, the month occurrence is sampled from a (discrete) $\operatorname{pdf} \mathcal{U}_{m}(\{1, \ldots, 12\})$, thus the $\eta_{\phi}^{l}$ are inferred from the corresponding pdf $\mathcal{N}_{m}\left(\mu_{m}, \sigma_{m}\right)$.

The sensor network consists of $N_{u}=11$ sensors, recording structural accelerations $\ddot{u}_{n}(t)$, and of $N_{\phi}=11$ thermometers, recording temperatures $\phi_{n}$, with $n=1, \ldots, 11$, arranged as depicted in Fig. 1 . We have considered dual output sensors, recording both accelerations and temperatures at the same location. The dynamical response is monitored with a sampling frequency of $20 \mathrm{~Hz}$, such as to sample the first two structural frequencies, respectively $2.79 \mathrm{~Hz}$ and $7.14 \mathrm{~Hz}$, without incurring in aliasing.

Thanks to the adoption of the ROM for the dataset construction, the number of dofs decreases from $M_{\varphi}=1469$ to 28 for the stationary diffusion problem and from $M_{v}=2938$ to 63 for the elasto-dynamic problem. Consequently, the CPU time required by each simulation, over the time interval $(0, T=35 \mathrm{~s})$, passes from $421 \mathrm{~s}$ to $4.9 \mathrm{~s}$, entailing a speed-up of about 86 times (computations have been run on a PC featuring an Intel (R) Core ${ }^{\mathrm{TM}}$, i5 CPU @ $2.6 \mathrm{GHz}$ and 8 GB RAM).

The evolution of the loss function and of the global accuracy of classifier $\mathcal{G}$, obtained during the training, are respectively reported in Figs. 2 and 3. The iteration number accounts for the number of times the FCN weights have been updated. The greatest gains in terms of classification accuracy (i.e., the ratio of correctly classified instances over the total) are obtained in the first portion of the graph.

The generalization capabilities of the classifier $\mathcal{G}$ have been assessed on a test set made of 108 pseudo-experimental instances generated through the FOM. The classifier $\mathcal{G}$ carries out the classification 
task with a global accuracy of $81.48 \%$ and a testing time of $1.08 \mathrm{~s}$, which means roughly about $0.01 \mathrm{~s}$ for each test instance. The obtained results are summarized by the confusion matrix in Fig. 4. Two different sources of error stand out. In particular, a small number of test instances labeled as $g=3$ are misclassified as $g=1$ (the same also occurs between $g=6$ and $g=3$ ); this might be due to the similar influence of those scenarios on the mechanical behavior. Moreover, half of the test instances labeled as $g=0$ (undamaged) are also misclassified; this might be due to the variability of $\delta$. Indeed, a low value of $\delta$ not only implies an augmented difficulty in distinguishing between damaged and undamaged conditions, but also causes the ROM to be less accurate.

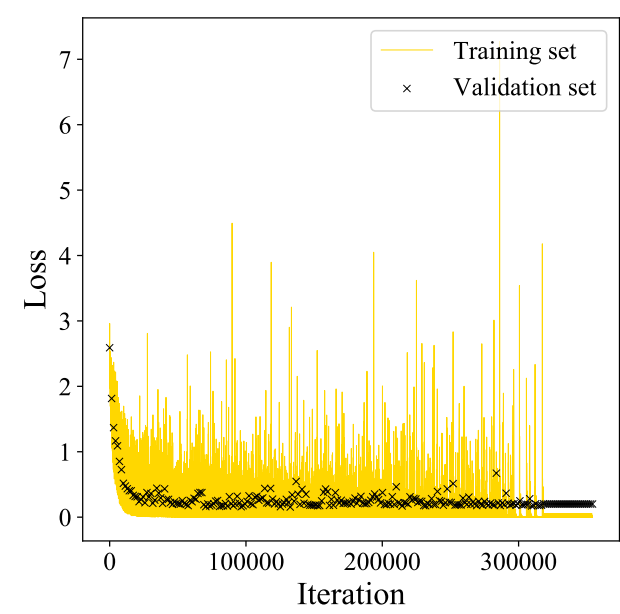

Figure 2. Classifier training: loss function evolution on the training and validation sets.

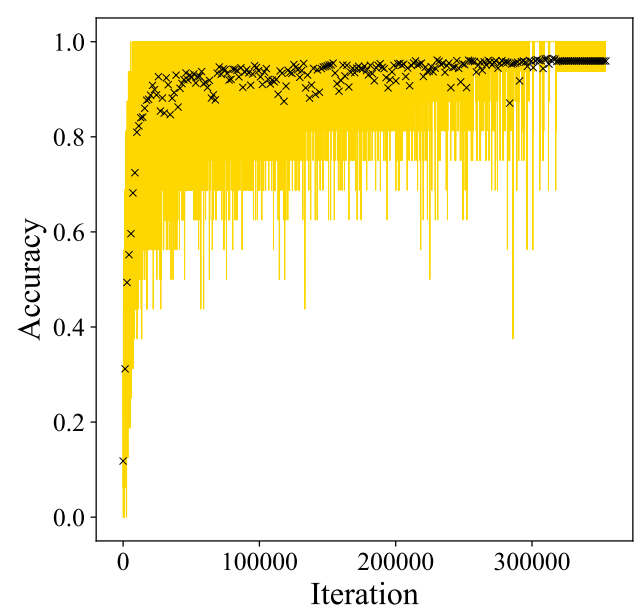

Figure 3. Classifier training: global accuracy evolution on the training and validation sets.

\section{Conclusions}

In this work, we have proposed a computational framework, integrating model-order reduction and deep learning, for structural health monitoring under varying operational and environmental conditions. This hybrid model-data strategy enables an online damage localization, making use of vibrational and temperature measurements. To overcome the lack of experimental data for civil applications, a database of synthetic recordings has been built offline, for a set of predefined damage scenarios, through simulations of a physics-based model, explicitly accounting for varying operational and environmental conditions. A parametric reduced order model, built through the reduced basis method, has been exploited to accelerate the dataset generation. Finally, a classifier exploiting a convolutional neural network has been adopted to perform automatic feature extraction and to relate raw sensor data to the corresponding structural health conditions.

In the presented example, the classification outcomes show a global accuracy of about $81 \%$, and offer the possibility to identify the nature behind the misclassification errors.

In future works, we aim to couple the classifier with a further neural network branch, playing the role of first line damage identifier, to reduce the possibility of an incorrect classification of undamaged scenarios. Moreover, to maximize the effectiveness for the damage assessment, a sensor placement according to a Bayesian optimization approach is going to be envisaged.

Acknowledgments: Prof. Alberto Corigliano (Politecnico di Milano) is gratefully acknowledged for the valuable support and its methodological inspiration.

Conflicts of Interest: The authors declare no conflict of interest.

\section{References}

1. Bigoni, C.; Hesthaven, J.S. Simulation-based Anomaly Detection and Damage Localization: an application to Structural Health Monitoring. Computer Methods in Applied Mechanics and Engineering 2020, 363, 112896. 


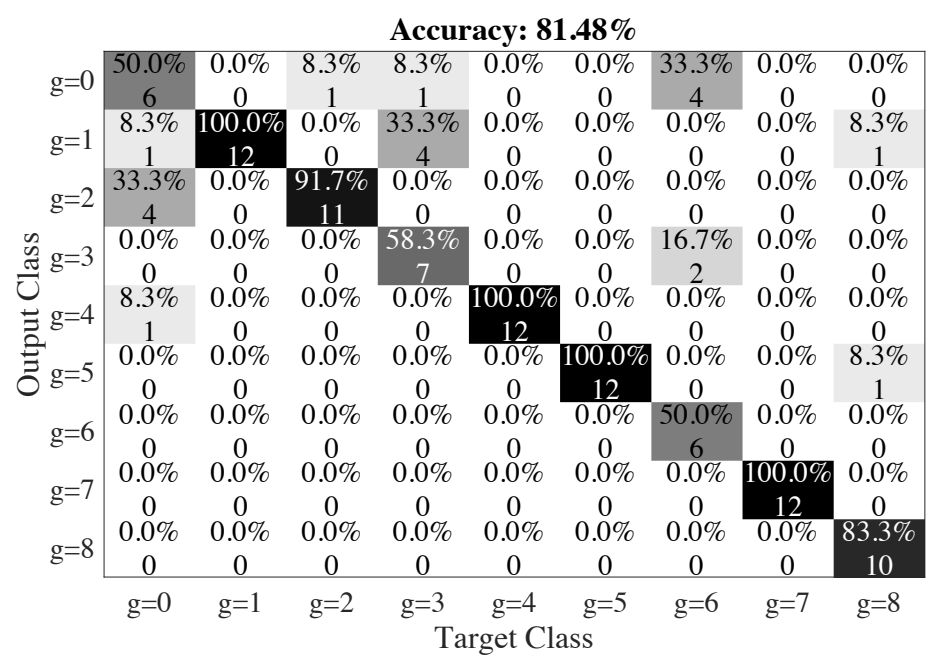

Figure 4. Classifier testing: confusion matrix.

2. Chang, P.C.; Flatau, A.; Liu, S.C. Review paper: health monitoring of civil infrastructure. Structural Health Monitoring 2003, 2, 257-267.

3. Eftekhar Azam, S.; Mariani, S. Online damage detection in structural systems via dynamic inverse analysis: A recursive Bayesian approach. Engineering Structures 2018, 159, $28-45$.

4. Sohn, H.; Worden, K.; Farrar, C.R. Statistical Damage Classification Under Changing Environmental and Operational Conditions. Journal of Intelligent Material Systems and Structures 2002, 13, 561-574.

5. Entezami, A.; Sarmadi, H.; Bhkamal, B.; Mariani, S. Big Data Analytics and Structural Health Monitoring: A Statistical Pattern Recognition-Based Approach. Sensors 2020, 20, 2328.

6. Fink, O.; Wang, Q.; Svensen, M.; Dersin, P.; Lee, W.; Ducoffe, M. Potential, Challenges and Future Directions for Deep Learning in Prognostics and Health Management Applications. Engineering Applications of Artificial Intelligence 2020, 92, 103678.

7. Taddei, T.; Penn, J.; Yano, M.; Patera, A. Simulation-based classification; a model-order-reduction approach for structural health monitoring. Archives of Computational Methods in Engineering 2018, 25, 23-45.

8. Rosafalco, L.; Manzoni, A.; Mariani, S.; Corigliano, A. Fully convolutional networks for structural health monitoring through multivariate time series classification. Adv. Model. and Simul. in Eng. Sci. 2020, 7, 38.

9. Rosafalco, L.; Corigliano, A.; Manzoni, A.; Mariani, S. Combined Model Order Reduction and Artificial Neural Network for data assimilation and damage detection in structures. Computational Sciences and Artificial Intelligence in Industry (CSAI), June 12-14, 2019. To appear.

10. Rosafalco, L.; Corigliano, A.; Manzoni, A.; Mariani, S. A Hybrid Structural Health Monitoring Approach Based on Reduced-Order Modelling and Deep Learning. Proceedings 2020, 42, 67-80.

11. Farrar, C.R.; Doebling, S.W.; Cornwell, P.; Straser, E.G. Variability of modal parameters measured on the Alamosa Canyon bridge. Proceedings of SPIE - The international society for optical engineering 1997, 1.

12. Eftekhar Azam, S.; Mariani, S.; Attari, N. Online damage detection via a synergy of proper orthogonal decomposition and recursive Bayesian filters. Nonlinear Dynamics 2017, 89, 1489-1511.

13. Corigliano, A.; Mariani, S. Parameter identification in explicit structural dynamics: Performance of the extended Kalman filter. Computer Methods in Applied Mechanics and Engineering 2004, 193, 3807-3835.

14. Sabetta, F.; Pugliese, A. Estimation of response spectra and simulation of nonstationary earthquake ground motions. Bulletin of the Seismological Society of America 1996, 86, 337-352.

15. Paolucci, R.; Gatti, F.; Infantino, M.; Smerzini, C.; Özcebe, A.G.; Stupazzini, M. Broadband ground motions from 3D physics-based numerical simulations using artificial neural networks. Bulletin of Seismological Society of America 2018, 108, 1272-1286.

(C) 2020 by the authors. Licensee MDPI, Basel, Switzerland. This article is an open access article distributed under the terms and conditions of the Creative Commons Attribution (CC BY) license (http:/ / creativecommons.org/licenses/by/4.0/). 